\title{
Les mécanismes de construction de mots en godié, langue kru de Côte D'Ivoire*
}

\author{
Damanan N'dré (Bouaké)
}

\begin{abstract}
This article analyzes the mechanisms of word building in Godié, a Kru language spoken in Côte d'Ivoire. Two processes underly these mechanisms: morphological and syntactic methods. Morphological processes refer to constructed words and syntactic processes refer to lexicalized syntactic sequences. At the morphological level, are generated by the constructed word by suffix and words constructed by juxtaposing two or three tokens. The language has three verbal

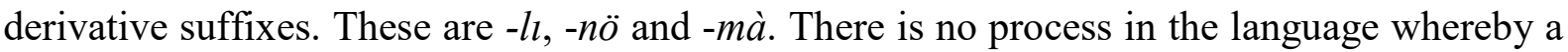
verb is derived from a noun. For words constructed by juxtaposition of tokens, there are generally three types obtained by. In other words, two or three nouns can combine to create new words; as a noun and an adjective can combine to create new words. As for new words, created by combining two or three names, it has been shown that the Internal underlying structure of bipartite type derived from a tripartite structure surface. However, the existence of the tripartite structure is allowed; but it emerges, within the language, from idiosyncratic phenomena. Even in lexicalized syntactic sequences, it was also observed that internal bipartite structure underneath. Moreover, between words constructed by combining three formants and lexicalized syntactic sequences, the limit is blurred. In the language, words constructed as syntactic lexicalized sequence may be a dictionary entry. Our study shows that the concept of a morphological right head is untenable. In the language, in fact, words may be constructed with both left and with right morphological heads. In addition, some words are constructed with morphological head whatsoever, as in many languages around the world.
\end{abstract}

\footnotetext{
${ }^{*}$ Le godié est une langue kru de Côte d'Ivoire (Marchese 1983 : 37). La langue comprend neuf dialectes totalement intercompréhensibles. Ce sont: le kagbowalé, le tigluwalé, le nugbowalé, le gnagowalé, le djrikowalé, le drogowalé, le gligbowalé, le koyo et le dadjriwalé. L'intercompréhension étant donc totale entre les dialectes de la langue, les items qui sont étudiés dans la présente étude sont connus de tous les locuteurs du godié. Mais l'étude tourne principalement autour du dadjriwalé (Godé 2008). C'est le dialecte situé à l'est de l'aire godié. Il est parlé par les locuteurs godié situés sur l'axe Lakota-Fresco. C'est le godié central, duquel dérivent tous les autres dialectes (Godé 2008 : 19). Les langues kru sont réparties en deux sous-groupes : occidental et oriental. Le groupe occidental comprend les langues guéré, grébo, wobé, etc. Le groupe oriental comprend les langues bété, dida, godié, etc. (Marchese 1989). Les langues kru sont rattachées, à l'intérieur du phylum Niger-Congo, au groupe nord Volta-Congo qui comprend aussi les langues gur et les langues Adamawa-Ubanguiennes (Williamson/Blench 2000).
} 


\section{$1 \quad$ Introduction}

La présente étude porte sur les mécanismes de construction des mots en godié. Elle consiste en une analyse de la structure interne des mots. Nous la situons dans l'approche morphologique basée sur le mot. Dans cette approche, c'est le mot, unité minimale de sens, qui est l'élément central de toute étude morphologique. On lui reconnait, contrairement au morphème, une structure phonologique. Il appartient également à une catégorie syntaxique et possède une valeur sémantique. Ces trois composantes fondent l'unité lexicale qu'incarne le mot. Lui seul « satisfait le critère du signe minimal », par opposition au morphème qui ne peut déterminer la forme phonologique d'un autre morphème à travers la frontière du mot. La thèse du mot, comme unité centrale de la morphologie, est défendue par Matthews (1974), Aronoff (1976), Corbin (1987), Anderson (1992), etc. Mais au sein de cette approche morphologique basée sur le mot, deux conceptions s'affrontent. En effet, certains comme Aronoff (1976) parlent de morphologie lexicale ; tandis que d'autres, comme Corbin (1987), parlent de morphologie constructionnelle. Les premiers partent de règles morphologiques pour analyser la formation de mots nouveaux. Pour les mêmes analyses, les seconds partent de schèmes morphologiques. Mais, que l'analyse soit basée sur des règles ou des schèmes morphologiques, pour caractériser la formation de mots nouveaux, il ne s'agit, qu'une question de terminologie. Car, si le schème morphologique $\left[[\mathrm{x}]_{\mathrm{V}} \text { er }\right]_{\mathrm{N}}$ 'one who Vs' de Booij (2008) correspond à la règle de morphologie lexicale $[\mathrm{x}]_{\mathrm{V}} \rightarrow\left[[\mathrm{x}]_{\mathrm{V}} \text { er }\right]_{\mathrm{N}}$, 'one who Vs habitually, professionally' d'Aronoff (1976), il ne peut s'agir à quelque exception près - que de la même chose (cf. Booij 2008). D'ailleurs, ce qui compte pour ces deux conceptions, c'est l'identification des éléments lexicaux, avec un intérêt particulier sur les relations de forme et de sens qu'entretiennent les constituants qui président à la construction de mots nouveaux. Ces deux conceptions tablent sur l'existence de deux catégories de mots complexes. Il y a, d'une part, les mots complexes construits et d'autre part, les mots complexes non construits. Un mot est dit construit, s'il est défini d'après une règle ou un schème morphologique. Il est dit non construit, si la règle ou le schème qui préside à sa construction découle de la syntaxe. De tels mots sont définis comme des séquences syntaxiques lexicalisées. La morphologie lexicale et/ou constructionnelle distinguent donc les composés morphologiques des séquences syntaxiques lexicalisées. Dans ce qui suit, il s'agira d'exposer les processus morphologiques et/ou syntaxiques qui président à la construction de mots nouveaux ou à la lexicalisation de séquences syntaxiques en godié. L'analyse consistera à identifier la structure des mots construits ou des séquences syntaxiques lexicalisées. Sans la situer dans un cadre morphologique particulier, elle entend emprunter, par endroit, le modèle théorique développé par Selkirk (1982). Nous exposerons brièvement, le modèle théorique choisi. Nous présenterons ensuite le cadre général des constructions lexicales complexes. Nous étudierons enfin les constructions morphologiques, puis les séquences syntaxiques lexicalisées.

\section{Le modèle théorique}

Dans la théorie X-barre, de laquelle théorie dérive le modèle théorique soutenu par Selkirk (1982), on considère que toute composante syntaxique obéit à des règles de structuration des unités linguistiques en phrase. De cette composante, toutes les phrases possibles d'une langue peuvent être dérivées. Il en est de même de la composante morphologique. Comme en syntaxe, en morphologie, il existe des règles de structuration des mots qui permettent de dériver autant de mots construits que peut permettre une langue. Toute unité lexicale complexe, quelle soit

ISSN 1615-3014 
dérivée ou non, obéit à des règles de formation. Elle a une structure interne qu'elle tient de ces règles de structuration. Qu'il s'agisse de la dérivation ou de la composition, toute structure morphologique est une sorte de structure syntaxique. Il ne s'agit donc pas d'une simple addition de constituants morphologiques. Nous considérons que la dérivation concerne la combinaison de mots et d'affixes, le tout formant des entités indissociables. Les affixes, bien qu'étant des morphèmes liés, sont considérés comme des mots, c'est-à-dire des catégories majeures. Dans la formation de mots dérivés, ce sont eux - les affixes - qui sont les têtes morphologiques, et " spécifiés en terme de sous-catégorisation ». Les bases, c'est-à-dire les mots auxquels ils s'adjoignent, sont considérées comme leurs compléments sous-catégorisés (cf. Brousseau 1989). Pour la composition, il s'agit d'une combinaison de mots. A ce niveau également, les têtes morphologiques - s'il en existe - sont spécifiées et les compléments sous-catégorisés sont appelés des modifieurs (Brousseau 1989). Dans l'une ou dans l'autre des formations de mots, il existe donc « des rapports de gouvernement entre des têtes et leurs compléments » (cf. Zwanenburg 1991).

Considérant que les structures à branchement binaire peuvent être appliquées aux unités lexicales complexes, on pourrait avoir, pour la dérivation et la composition, les deux structures arborescentes respectives suivantes : 1

(1a)

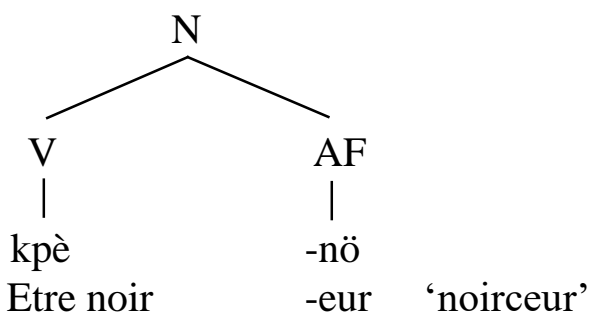

(1b)

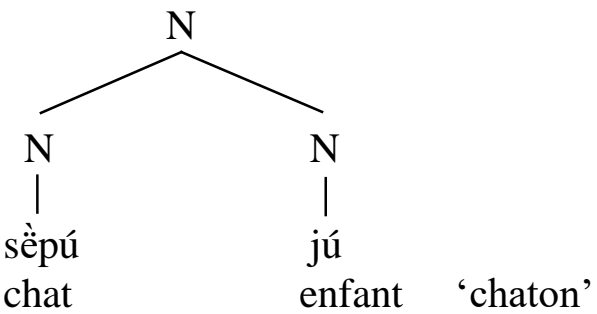

Dans chacune de ces deux représentations, l'essentiel de l'information sémantique est porté par le constituant de droite, considéré à ce titre comme la tête du mot construit. C'est au moyen de la percolation que s'effectue cette information sémantique. En effet, comme dit Brousseau $(1990: 29)$ : «le transfert des informations sémantiques et syntaxiques [...] des constituants d'un mot à ce mot est opéré au moyen de la percolation ». L'auteur définit la percolation comme suit :

a.) Si une tête spécifiée pour un trait $[\alpha \mathrm{Fj}], \alpha \neq$ non-spécifié, le nœud-mère de cette tête doit être spécifié $[\alpha \mathrm{Fj}]$.

b.) Si une non-tête est spécifiée pour le trait $[\beta F j]$ et que la tête est non-spécifiée pour ce trait, le nœud-mère doit être spécifié $[\beta F j]$

\footnotetext{
${ }^{1}$ Nos données ont été collectées auprès d'un locuteur du dadjriwalé. Mais elles ont été testées auprès d'autres locuteurs Godiés pour vérifier l'intercompréhension entre les différents locuteurs Godiés. Ce fut un excellent test, car l'intercompréhension est quasiment totale. Pour ce qui est de la transcription des données, nous nous sommes inspirés de l'orthographe harmonisée des langues kru (cf. Kipré Blé et al. 2013). Toutefois, certaines voyelles ont été transcrites par nous telles qu'elles existent phonétiquement. Il s'agit des voyelles $[1],[\varepsilon],[\varpi]:$ alors qu'elles

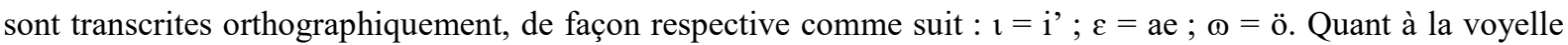
postérieure -ATR mi-ouverte [0], elle a pour correspondant orthographique ao. C'est cette voyelle qui correspond dans nos transcriptions à la voyelle ö. Il est à remarquer également que seuls les tons haut et bas sont notés. Le ton moyen ne l'est pas. Il est considéré comme un ton neutre, donc non marqué.
} 
Dans nos exemples en (1), le dérivatif -nö et le constituant nominal jú 'enfant', en tant que porteurs essentiels de l'information sémantique, déterminent la catégorie à laquelle appartiennent les mots construits, respectivement. La détermination de cette catégorie est plus marquée dans les constructions dérivatives que dans la composition.

Suivant la thèse défendue par Williams (1981), Selkirk (1982), Lieber (1981), Di Sciullo et Williams (1987), " la tête d'un mot construit est le constituant le plus à droite ». Les exemples en (1) justifient cette thèse. Selon ces auteurs, la thèse de «tête morphologique à droite » tient lieu de règle universelle. Autrement dit, elle se justifierait, dans toutes les langues du monde, comme une condition sine qua non dans la formation de mots complexes. Mais cette thèse est battue en brèche par l'exemple suivant :

(2)

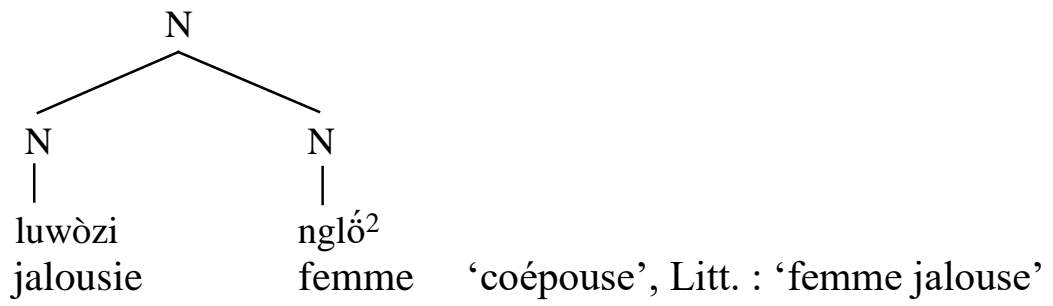

Comme nous pouvons le constater, dans cette structure arborescente, l'essentiel de l'information est porté par le constituant de gauche. C'est ce constituant qui en est la tête ; et le constituant de droite en est le modifieur. La notion de tête morphologique ne répond donc pas à des lois universelles. Elle semble liée à des paramètres propres à chaque langue (Payne 1997 ; Fabb 2001). L'idée que la tête en structure morphologique n'est pas toujours à droite est partagée par nombre d'auteurs (Brousseau 1989, 1990 ; Zwanenburg 1991). Les différentes structures arborescentes ci-dessus montrent qu'en godié, la tête morphologique peut être à droite ou à gauche. Comme en godié, dans beaucoup de langues, cette possibilité de tête morphologique à droite et à gauche existe (Booij 2007). Des auteurs comme Corbin (1987) n'admettent pas la notion de " tête morphologique " comme l'élément crucial dans l'analyse des faits morphologiques. D'ailleurs, certaines constructions morphologiques, dans de nombreuses langues, militent en faveur de cette position de Corbin. En godié par exemple, il existe des constructions morphologiques considérées comme n'ayant pas de tête morphologique. Il s'agit - dans le cas d'espèce - de constructions morphologiques dont les constituants appartiennent à des classes grammaticales différentes. Ainsi, on ne peut parler de tête morphologique dans la structure arborescente ci-dessous :

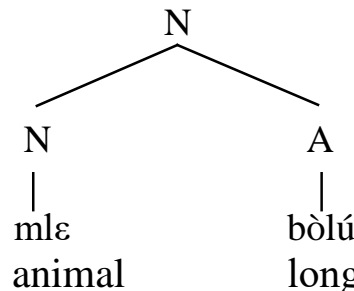

\footnotetext{
${ }^{2}$ Les exemples en (1b) et en (2) sont structurellement les mêmes. Se sont des syntagmes génitivaux à juxtaposition $\mathrm{N} 1+\mathrm{N} 2$. Mais les deux exemples diffèrent par leur sémantisme. En (1b), N1 est un hypéronyme. En (2), c'est N2 qui a cette valeur. Les noms qui leur sont adjoints permettent d'obtenir des hyponymes. Ainsi, les deux syntagmes génivaux pourraient être glosés en français, respectivement par : 'Le petit de chat' pour sềpú-ju et 'Femme de jalousie‘ pour luwòzì-nglö. Ceci indique que ce sont N2 en (1b) et N1 en (2) qui sont les déterminants, les porteurs de sens donc les têtes morphologiques respectivement. Dans le premier, la tête morphologique est à droite ; dans le second, elle est à gauche.
} 
Ici, le constituant de droite - qui porte l'essentiel de l'information sémantique - n'appartient pas à la même catégorie grammaticale que le constituant de gauche. L'un, mle (animal) appartient à la catégorie des noms ; l'autre, bòlú (long) appartient à la catégorie des adjectifs. Dans de tels cas, on ne parle plus de tête morphologique (cf. Brouseau 1990).

Mais la notion de tête morphologique implique des relations d'inclusion qu'entretient l'hyponymie. L'hyponymie est définie, d'après Brousseau (1990 : 28-29) : " comme la relation entre $\mathrm{X}$ (e. g. meuble) et des mots Y, Z, W, plus spécifiques (e. g. table, bureau, vaiselier), où l'intension de $\mathrm{X}$ est incluse dans les intensions de tous les mots $\mathrm{Y}, \mathrm{Z}, \mathrm{W}$, ses hyponymes ». Relativement à cette définition, on pourrait considérer en (2), que le lexème luwòzì-nglö 'coépouse' est en relation d'inclusion avec le lexème nglö 'femme'. Autrement dit, luwozi-nglö 'coépouse' est un sous-ensemble de nglö 'femme'. Il en est de même du mot construit 'homme-orchestre' où 'l'ensemble des hommes-orchestres' est un sous-ensemble de celui des hommes .3 «Homme-orchestre » comme luwòzì-nglö 'coépouse' sont des hyponymes respectifs des constituants 'homme' et nglö 'femme'.

L'une des raisons qui ont motivé le choix du modèle théorique de Selkirk (1982) est surtout celle de la structure binaire comme l'indiquent les différentes représentations ci-dessus. Dans la langue, en dehors de quelque constructions idiosyncrasiques, toute construction morphologique ou syntaxique - quel que soit le nombre de ses constituants - semble obéir à la structure binaire (cf. infra).

\section{Généralité sur les unités lexicales complexes}

Les mécanismes de construction de mots en godié sont très complexes. Certaines constructions associent deux bases lexicales; quand d'autres en associent trois, voire quatre. Dans celles où sont mises en association deux bases, il peut s'agir de deux noms, d'un nom et d'un adjectif ou d'un nom et d'un verbe. Ce mécanisme, qui consiste à associer deux unités pour créer un mot nouveau, est de loin le plus simple et le plus productif dans la langue. Le plus complexe des mécanismes consiste en l'association de plus de deux unités dans la formation de mots nouveaux. Dans ce type de construction, la possibilité de création d'unités lexicales nouvelles par la réduplication d'un verbe associé à un nom existe. Aussi, avec les verbes de locution, en association avec un nom pour créer de nouvelles unités lexicales, la base verbale est prise en étau entre sa partie nominale et le nom qui lui est associé. On observe ainsi, en l'espèce, une sorte de métathèse avec l'antéposition du nom comme élément central du mécanisme de construction de mots nouveaux à partir des verbes de locution ou des verbes à objets inhérents.

Mais quel que soit le type de mécanisme mis en jeu, la structure interne de la nouvelle unité lexicale obtenue est de l'ordre « déterminant + déterminé » ou « déterminé + déterminant ». Ces deux types de structures sont proches - respectivement - de celles du syntagme génitival et du syntagme épithétique. Il va sans dire que, dans la construction de mots nouveaux, l'élément qui gouverne peut être de gauche ou de droite, en rapport avec le type de structure mis en contribution. Mais à la différence du syntagme génitival et du syntagme épithétique, dans les mécanismes de création de mots nouveaux, les termes associés par juxtaposition aliènent leurs

\footnotetext{
${ }^{3}$ L'exemple est tiré de Brousseau (1990 : 29).
} 
signifiés initiaux (cf. Godé 2008). Ils en acquièrent de nouveaux qui sont plus ou moins différents de ceux dont ils sont les dérivés.

En effet, dans le cas de constructions génitivales ou épithétques, il ne s'agit pas d'un recouvrement de signifié nouveau, mais d'une restriction du signifié du terme déterminé. De telles constructions sont l'expression d'une caractérisation. De plus, ces constructions peuvent être élargies par l'addition - à chaque formant de la structure déterminative - d'une unité lexicale ou grammaticale. Ce n'est pas le cas d'une construction compositionnelle ou dérivationnelle. En effet, les substantifs obtenus par dérivation ou par composition sont caractérisés par le figement des relations internes des constituants. Autrement dit, on ne peut insérer à l'intérieur des formants d'une unité dérivée et/ou composée une autre unité linguistique susceptible de les élargir. L'inséparabilité des formants de l'unité dérivée ou composée est l'un des critères fondamentaux de la distinction entre mots composés et groupes syntaxiques (Corbin 1992 et 1997). ${ }^{4}$ C'est sur la base de cette distinction que nous analyserons, dans ce qui suit, les différentes constructions de mots complexes dans la langue. Nous opérons ainsi, grossièrement, dans les constructions de mots complexes, deux grandes subdivisions. Il s'agit, d'une part, des constructions morphologiques et, d'autre part, des constructions syntaxiques lexicalisées.

\section{$4 \quad$ Les constructions morphologiques}

Nous distinguons deux types de constructions morphologiques. Il s'agit des constructions morphologiques par affixation d'un dérivatif à l'unité lexicale de base et des constructions morphologiques par association de deux ou de plus de deux unités lexicales.

\footnotetext{
${ }^{4}$ Le critère même de l'inséparabilité, comme paramètre de distinction entre mots construits et groupes syntaxiques nous semble peu défendable. Ce critère reste tout de même flou dans la langue. On a du mal, par exemple, à considérer les unités lexicales complexes issues de locutions verbales comme des mots construits ou des séquences syntaxiques lexicalisées. Il existe également des expressions lexicales qui acceptent les deux interprétations morphologique et/ou syntaxique. Dans la langue, l'expression sáká sèní en est une illustration :
}

(1) sáká sèní riz seaux

Elle peut être analysée comme un mot construit ou comme un groupe syntaxique. Comme groupe syntaxique, on peut insérer entre les deux constituants, le connectif lá qui, soulignons-le, reste tout de même facultatif. Ce connectif véhicule les valeurs sémantiques de la préposition de ou pour du français. Observons :

(2) Sáká (lá) sèní

riz CON seaux

'des seaux de riz' ou 'des seaux pour riz'

Ce syntagme géntival pluriel donne au singulier :

(3) Sáká (lá) sònú

riz CON seau

'un seau de riz » ou « un seau pour riz'

Mais l'expression sáká sèní, en tant que mot construit, désigne un aliment et peut être traduit en français par « soupe de riz » ou « bouillie de riz » communément appelé baka dans le jargon ivoirien. Comme tel, elle constitue un bloc indissociable. On ne saurait insérer entre les deux constituants sáká 'riz' et sèní 'seaux' un élément susceptible d'élargir le signifié de l'expression sáká sèni 'soupe de riz'.

A l'instar du godié, certaines langues ont ces expressions morphologiques qui peuvent être analysées comme composés ou comme groupes syntaxiques. C'est le cas de la suite de mot anglais glass case qui identifie une vitre d'une part et un coffre en verre d'autre part (cf. Jespersen (1911, VI, 135) cité par Bassac (2003 : 200).

ISSN 1615-3014 


\subsection{Les constructions par affixation d'un dérivatif}

La langue connaît deux morphèmes de dérivation permettant de substantiver des verbes. Il s'agit des suffixes $-l l$ et $-n \ddot{o}$. Le premier est de loin le plus productif.

\subsubsection{La substantivation par le dérivatif -lı}

Le dérivatif -lı est très productif. La plupart des verbes - sinon la quasi-totalité - sont substantivés à partir de ce dérivatif. Avec les bases verbales simples, la suffixation est plus aisée :

(4)

$\begin{array}{llll}{[\mathrm{x}]_{\mathrm{V}}} & & \left.[\mathrm{x}]_{\mathrm{V}}-\mathrm{ll}\right]_{\mathrm{N}} \\ \text { plaà } & \text { 'maudire' } & & \text { plaà-lı } \\ \mathrm{m} \omega & \text { 'aller, partir' } & & \text { m } \omega-1 \mathrm{l}\end{array}$

'le fait de maudire, malédiction' 'le fait de partir, départ'

Avec les bases verbales complexes, on observe une permutation des constituants. Ainsi, dans la substantivation des locutions verbales, le constituant nominal est antéposé, permettant la base verbale de recevoir le suffixe dérivatif $-1 \iota$ :

$$
\begin{array}{llll}
{[\mathrm{x}]_{\mathrm{V}}} & \rightarrow & \left.[\mathrm{x}]_{\mathrm{V}}-1 \mathrm{l}\right]_{\mathrm{N}} & \\
\text { pá mesí } & \text { 'prédire' } & & \\
\text { pá nyú pà-lı } & \text { 'bénir' } & & \text { nyu pà-lı }
\end{array} \quad \text { 'le fait de prédire, prédiction, prophétie' }
$$

\subsubsection{La substantivation par le dérivatif -nö}

Le dérivatif -nö ne permet de substantiver que des verbes spécialisés dans l'expression de la caractérisation. Il s'agit de verbes que l'on pourrait qualifier de verbes-adjectifs. Il en existe de simples, comme il en existe de complexes, à l'instar des verbes « réguliers ».

\begin{tabular}{|c|c|c|c|}
\hline$[\mathrm{x}] \mathrm{V}$ & & {$\left[[\mathrm{x}]_{\mathrm{V}}-\mathrm{nö}\right]_{\mathrm{N}}$} & \\
\hline ngö kẃ & 'être lourd' & 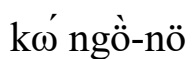 & 'lourdeur' \\
\hline tl $\omega^{\prime} \mathrm{k} \omega$ & 'être long' & $k \omega^{\prime}$ tl⿳亠丷⿵冂⿱丷口心 & 'longueur' \\
\hline wo klá & 'être large' & kl $\omega$ wò-nö & 'largeur' \\
\hline
\end{tabular}

Avec les verbes- adjectifs simples, on a les constructions suivantes :

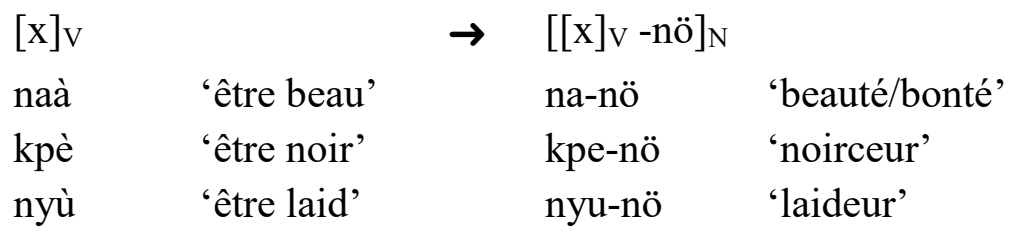

Avec les verbes- adjectifs complexes, on a les constructions suivantes :

Comme dans les verbes « réguliers » complexes, ici également, on observe le même phénomène : la permutation des constituants. En l'espèce, la particule postposé dans la locution verbale, s'antépose, laissant libre cours au radical verbal de recevoir le dérivatif -nö.

En dehors du phénomène de substantivation de verbes par les dérivatifs -lı et -nö, la langue connaît un autre dérivatif fonctionnant comme un morphème de complexification ou de recomplexification de base verbale. C'est le dérivatif -mà.

\subsubsection{Complexification d'items verbaux par le dérivatif -mà}

Le dérivatif -mà permet d'obtenir des verbes transitifs à partir des verbes-adjectifs. Observons : 
(8)

$\begin{array}{lllll}{[\mathrm{x}]_{\mathrm{V}}} & & \rightarrow & \left.[\mathrm{x}]_{\mathrm{V}}-\mathrm{mà}\right] \mathrm{V} & \\ \text { naà } & \text { 'être beau' } & & \text { na-mà } & \text { 'embélir, rendre beau' } \\ \text { kpè } & \text { 'être noir' } & & \text { kpe-mà } & \text { 'noircir, rendre noir' } \\ \text { nyù } & \text { 'être laid' } & & \text { nyu-mà } & \text { 'enlaidir, rendre laid' }\end{array}$

Comme nous pouvons le constater, -mà est un dérivatif verbal à valeur de causatif ou d'auxiliaire factif N'dré $(2011,2012)$. Il pourrait être traduit en français par le verbe rendre qui synthétise, d'après Gross (1981), le causatif « faire devenir ».

Remarque : Ces verbes obtenus à partir du suffixe causatif -mà, peuvent à leur tour, être substantivés par le dérivatif -lı:

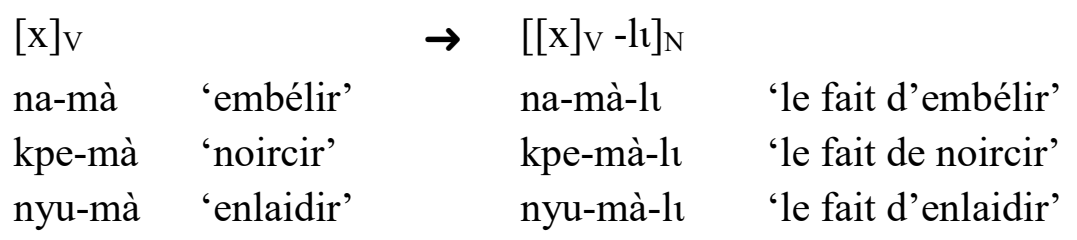

Il ressort de cette remarque que le dérivatif -lı est un véritable morphème de nominalisation.

NB : Il n'existe pas dans la langue de morphème dérivatif qui permettrait de construire des verbes à partir de noms.

\subsection{Les constructions par association de bases lexicales}

\subsubsection{Les constructions à deux bases lexicales}

Les mécanismes de construction de nominaux à partir de deux bases est très productif en godié. Certaines constructions associent deux bases lexicales. D'autres constructions associent une base lexicale et une base adjectivale. Enfin, il existe des constructions dans lesquelles sont associées un verbe et un nom.

\subsubsection{Les constructions de type : $N+N$}

Le mécanisme d'association de deux noms pour créer des mots nouveaux est très productif en godié. Pour ce type de mots, les noms associés, par juxtaposition, aliènent leurs signifiés initiaux, et en acquièrent de nouveaux, plus ou moins différents des précédents. Toutefois, il arrive que le signifié du mot construit soit déductible des signifiés des noms en association. La structure interne $[\mathrm{N}-\mathrm{N}]_{\mathrm{N}}$ des unités en association peut être de l'ordre « déterminant+déterminé » ou de l'ordre « déterminé+déterminant». C'est de ce point de vue, qu'est déduite l'idée selon laquelle, l'élément qui gouverne, dans telles structures, est soit de gauche, soit de droite. Cidessous, sont illustrés des mots construits par association de deux noms :
a. $\mathrm{su}+$ dolu
(arbre + sang)
$\rightarrow$ su-dòlu ${ }^{5}$
'sève'
sáká + vá
(riz + barbe)
$\rightarrow$ sáká-va
'gerbe de riz'

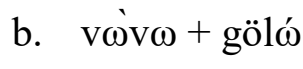
(vent + pirogue $)$

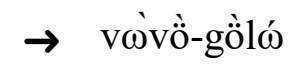
'bateau à voile'

\footnotetext{
${ }^{5}$ Le ton moyen n'est pas noté. Nous le considérons comme un ton neutre. Ainsi, l'absence de marque tonale sur un item ou une syllabe doit être considérée comme une trace du ton moyen.
} 


\begin{tabular}{|c|c|c|c|}
\hline $\mathrm{d} \omega \grave{d} \omega+$ nö & (terre + asticot $)$ & 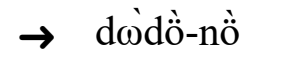 & 'larve de terre' \\
\hline pátá + kofế & (vide + trou $)$ & pàtà-kòfế & 'hangar' \\
\hline bùdū + kofé̀ & $($ trait + trou $)$ & $\rightarrow$ bùdù-kòfế & 'piste' \\
\hline làgö + dồbhé & $($ dieu + hache $)$ & làgồ-dồbhé & 'foudre, pierre esp.' \\
\hline kòsu + láka & $($ feu + caisse $)$ & $\rightarrow$ kòsù-làka & 'boîte d'allumettes' \\
\hline
\end{tabular}

On observe des modifications tonales dans ces mots construits. Ainsi, dans les items en (10a), les modifications concernent le deuxième constituant du terme construit. La syllabe en contact direct reçoit un abaissement tonal. Si le ton de cette syllabe est un ton haut, il devient ton moyen ; s'il est moyen, il devient bas. Les premiers constituants ne sont pas touchés par ces modifications tonales. En revanche, dans les items en (10b), les modifications sont aussi bien d'ordre tonal que vocalique. En effet, le segment vocalique [ $\omega]$ de la syllabe finale dans les constituants $v \omega v \omega$ 'vent' et $d \omega d \omega$ 'terre' est substitué par le segment vocalique [ö] de la syllabe immédiatement adjacente. Il s'agit, en l'espèce, d'une assimilation vocalique régressive. En ce qui concerne les modifications tonales, dans ces mots construits, on observe un abaissement tonal. L'abaissement tonal concerne les voyelles des syllabes immédiatement adjacentes. Le ton moyen qu'elles portent, en isolation, est abaissé d'un cran. En (10c), les modifications tonales concernent le premier constituant, plus ou moins dans son entièreté et la syllabe immédiatement adjacente du deuxième constituant nominal. En (10d), seule la voyelle du dernier constituant syllabique subit un abaissement tonal. Du registre moyen, elle passe au registre bas.

Dans les mots construits en (11), ci-dessous, on observe une troncation de la voyelle ou de la syllabe directement en contact avec le deuxième constituant du terme construit :

\begin{tabular}{|c|c|c|c|}
\hline nyíé ${ }^{6}+$ gbżle & (eau + rhinocéros) & $\rightarrow$ nyí-gbèlé & 'hippopotame' \\
\hline nyíé + zëbë & (eau + tortue esp.) & nyí-zëbë & 'tortue des rivières' \\
\hline nyíé + gbë & $($ eau + enclos $)$ & nyí-gbë & 'rivage' \\
\hline $\mathrm{Jie}+1 \varepsilon$ & (mer + éléphant) & $\mathrm{J} \tilde{1}-1 \varepsilon$ & 'baleine' \\
\hline $\mathrm{zabh} \omega+\mathrm{su}$ & (noix de coco + arbre) & za-su & 'cocotier' \\
\hline
\end{tabular}

En dehors du phénomène de troncation, dans le premier mot construit, on observe une assimilation vocalique progressive. Sous l'influence de la voyelle fermée +ATR [i], la voyelle $[\varepsilon]$, du constituant nominal gbèle 'rhinocéros', connait une élévation vocalique. La voyelle $[\varepsilon]$ est donc

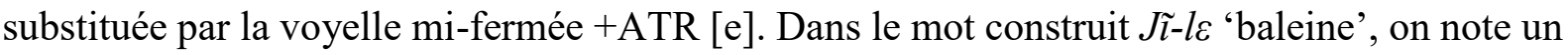
trait de nasalité. Ce trait de nasalité a une valeur locative. $J \tilde{l}-l \varepsilon$ 'baleine' signifie, de façon littérale, 'éléphant de mer'.

Dans certains mots construits, le deuxième formant semble avoir deux valeurs sémantiques différentes. Soit les items ci-dessous :

$$
\begin{array}{lll}
\text { a. } & \text { goji }+ \text { jú } & \text { (chien }+ \text { enfant }) \\
\text { sềpú }+ \text { jú } & \text { (chat }+ \text { enfant })
\end{array}
$$$$
\begin{array}{ll}
\rightarrow \text { gojì-ju } & \text { 'chiot' } \\
\rightarrow \text { sềpú-ju } & \text { 'chaton' }
\end{array}
$$

\footnotetext{
${ }^{6}$ Le terme nyié signifie en réalité une étendue d'eau. En dehors du koyo qui désigne tout étendu d'eau par nyi, les autres dialectes du godié le désignent par nyié.
} 


$\begin{array}{lllll}\begin{array}{lll}\text { bhábhlé }+ \text { jú } \\ \text { lo }+ \text { jú }\end{array} & \begin{array}{l}\text { (mouton }+ \text { enfant) } \\ \text { (éléphants }+ \text { enfant) }\end{array} & \text { bhábhli-ju } & \begin{array}{l}\text { 'agneau' } \\ \text { 'éléphanteau' }\end{array} \\ \text { b. lowluú }+ \text { jú } & \text { (à l'étranger }+ \text { enfant) } & \rightarrow \text { lowù-ju } & \text { 'allochtone' } \\ \text { gòJe }+ \text { jú } & \text { (godié }+ \text { enfant) } & \rightarrow \text { gòJè-ju } & \text { 'un godié' } \\ \text { láJá }+ \text { jú } & \text { (alladian }+ \text { enfant) } & \rightarrow \text { láJá-ju } & \text { 'un alladian' }\end{array}$

On observe qu'en (12a), le formant de droite jú 'enfant' attribue une valeur diminutive au mot construit avec le sens précis de 'petit'. Ici, la base nominale désigne un animal. En (12b), jú 'enfant' attribue une valeur locative au mot construit avec le sens de 'originaire de'. De ces constructions, deux règles morphologiques peuvent être déduites respectivement :
a. $\left[[+\mathrm{N},-\mathrm{V}]_{[+ \text {diminutif]-jú }]_{\mathrm{N}}}\right.$
b. $\left[[+\mathrm{N},-\mathrm{V}]_{[+ \text {locatif]-jú }]_{\mathrm{N}}}\right.$

Par analogie à ces deux règles, on pourrait concevoir la règle suivante :

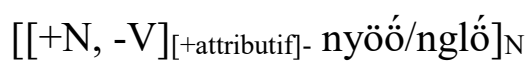

Elle permettrait, nous semble-t-il, de rendre compte des mots construits ci-dessous :

$$
\begin{array}{lllll}
\text { luwòzi + nglố } & \text { (jalousie }+ \text { femme }) & \rightarrow \text { luwòzì-nglö } & \text { 'coépouse' } \\
\text { kpakplá + nyöö } & \text { (mensonge + personne) } & \rightarrow \text { kplàkplì-nyö } & \text { 'menteur' } \\
\text { kplakplá + nglố } & \text { (mensonge + femme) } & \rightarrow \text { kplàkplì-nglö } & \text { 'menteuse' }
\end{array}
$$

Ici, les nominaux nyöó et nglö confèrent aux mots construits une valeur attributive.

Toutes ces unités lexicales complexes, ci-dessus, sont issues, chacune, de la juxtaposition de deux substantifs. Leurs sens, comme nous l'avons dit, sont déductibles de ceux des unités dont elles sont les composées. Leurs constituants étant issus de la même classe grammaticale, ces unités lexicales complexes sont appelées «composées endocentriques ». Dans de tels composés, l'un des constituants est défini comme tête et l'autre comme modifieur de tête (cf. Bisetto/Scalise 2005 ; Scalise/Guevara 2006). Dans les items en (10), tous les constituants de droite sont des têtes morphologiques et les constituants de gauche sont considérés comme des modifieurs (Di Sciullo/Williams 1987). En revanche, dans les items en (11), en (12) et en (15), c'est plutôt les constituants de gauche qui semblent jouer le rôle de tête morphologique et les constituants de droite celui de modifieur.

Comme nous l'avons souligné (cf. supra), tout composé endocentrique réfère à une sous-classe de la tête lexicale. Le mot construit dògòbhì-su 'citronnier', par exemple, est une sous-classe du lexème $s u$ 'arbre'. Autrement dit, un composé endocentrique est un hyponyme du constituant-tête, considéré, lui, comme un hypéronyme et spécifié, en l'espèce, par le référent du constituant-modifieur. Seuls les mots construits en (10) obéissent à la «Right-Hand Head Rule » de Williams (1981), Di Sciullo/Williams (1987). Dans les mots construits en (11), (12) et (15), la « Right-Hand Head Rule » est violée. Il va sans dire que dans la langue, la tête morphologique d'un mot construit peut être à droite ou à gauche. ${ }^{7}$

\footnotetext{
${ }^{7}$ La notion de tête lexicale est une invention des théories syntaxiques anglo-saxonnes. Dans ces théories syntaxiques, les analyses des phénomènes morphologiques sont basées sur la notion de tête lexicale. Ces analyses s'inspirent de la théorie $\mathrm{x}$-barre et du fonctionnement syntaxiques des constituants de la phrase. Est définie comme
} 


\subsubsection{Les constructions de type : $\mathrm{N}+\mathrm{A}$}

Les unités lexicales complexes ci-dessous illustrent le type de mots construits à partir d'un nom associé à un adjectif :

$$
\begin{array}{llll}
\text { gla }+ \text { dìdli } & \text { (dents }+ \text { lisse }) & \rightarrow \text { gla-dìdli } & \text { 'gencives' } \\
\text { ml } \varepsilon+\text { bòlú } & \text { (animal }+ \text { long) } & \rightarrow \text { mlë-bòlú } & \text { 'serpent' } \\
\text { gèsé }+ \text { fốkố } & \text { (fil }+ \text { léger }) & \rightarrow \text { gèsé-fốkố } & \text { 'coton' } \\
\text { jú }+ \text { dồdö } & (\text { enfant }+ \text { précoce }) & \rightarrow \text { jú-dồdö } & \text { 'bébé, nourrisson' }
\end{array}
$$

Ces unités lexicales complexes sont généralement qualifiés de composés exocentriques par opposition aux composés qualifiés d'endocentriques. La dichotomie composés endocentriques/composés exocentriques est adoptée par des auteurs tels que Williams (1981), Spencer (1991), Fabb (2001), Haspelmath (2002), Plag (2003), Booij (2005), Bisetto/Scalise (2005). Dans les composés exocentriques, les constituants de l'unité complexe construite appartiennent à des classes grammaticales différentes. Ici, c'est un adjectif qui s'associe à un nom pour former une unité lexicale complexe. Alors que dans les composés endocentriques, les constituants de l'unité complexe construite appartiennent à la même classe grammaticale. Les composés exocentriques sont considérés comme n'ayant pas de tête morphologique. Dans les items ci-dessus, les constituants de droite peuvent s'interpréter comme les qualifiants des noms auxquels ils sont adjoints ; mais ils n'ont pas la possibilité d'être des têtes sémantiques. Comme le dit si bien Lieber $(1992: 82)$ : « [...] exocentric compounds are those in which either semantically or syntactically (or both), the compound as whole does not bear the characteristics of its head». La structure de tels mots construit répond à celle du syntagme épithétique dont l'ordre est « déterminé+déterminant » ou « qualifié+qualifiant $» .8$

A coté de ces unités lexicales complexes obtenues par la juxtaposition d'un nom et d'un adjectif, nous pouvons citer le cas d'unités complexes obtenues par l'addition à l'unité de base, d'une unité linguistique mettant en relief l'opposition de sexe. Les unités linguistiques qui mettent en évidence cette opposition sont au nombre de deux. Il s'agit des unités lexicales bhéló et gá signifiant respectivement : 'mâle' et 'femelle'. Illustrent ce type de noms, les exemples ci-dessous : ${ }^{9}$

$$
\begin{array}{llll}
\text { bhábhlé + bhél } & \text { (mouton + mâle) } & \rightarrow \text { bhábhlí-bhèl } \omega & \text { 'bélier' } \\
\text { bhábhlé + gá } & \text { (mouton + femelle) } & \rightarrow \text { bhábhlí-ga } & \text { 'chèvre' }
\end{array}
$$

\footnotetext{
« tête » d'une unité morphologique ou syntaxique tout constituant ayant la capacité de transmettre ses propriétés catégorielles, syntaxiques et sémantiques à l'unité lexicale construite par composition ou par dérivation. Dans les composés exocentriques, aucun des constituants rentrant dans leur formation n'a cette capacité. D'après William (1981: 247-248), la tête d'une unité morphologique, qu'il s'agisse d'un mot dérivé ou composé, se situe à droite. ${ }^{8}$ Mais à la différence du syntagme épithétique dont les constituants peuvent être élargis par l'insertion d'autres unités linguistiques, les mots construits, par l'association d'un nom et d'un adjectif, sont caractérisés par le figement des relations internes. Autrement dit, il est impossible d'insérer à l'intérieur des composants de l'unité lexicale ainsi formée, une autre unité linguistique. Ainsi, contrairement au syntagme épithétique, dans le mot construit, le lien entre le nom et l'adjectif est très étroit. C'est ce lien étroit qui permet d'analyser l'unité formée par la juxtaposition du nom et de l'adjectif, non plus comme une unité épithétique, mais plutôt comme un composé lexical.

${ }^{9}$ Les unités lexicales bhéló 'mâle' et gá 'femelle' marquent l'opposition de sexe et concernent toutes les espèces, autre que l'espèce humaine.
} 


\begin{tabular}{|c|c|c|c|}
\hline 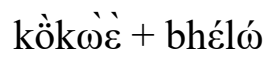 & (poulet + mâle) & $\rightarrow$ kö̀köö-bhèl $\omega$ & 'coq' \\
\hline kồkஸ̀غ + gá & (poulet + femelle) & $\rightarrow$ kòköố-ga & 'poule' \\
\hline goji + bhéló & (chien + mâle $)$ & gojì-bhèl $\omega$ & ‘chien’ \\
\hline soji + gá & $($ chien + femelle $)$ & $\rightarrow$ gojì-ga & 'chienne' \\
\hline èpú + bhéló & (chat + mâle $)$ & $\rightarrow$ sềpú-bhغ̀l $\omega$ & 'chat' \\
\hline èpú + gá & (chat + femelle $)$ & $\rightarrow$ sềpú-ga & 'chatte' \\
\hline
\end{tabular}

\subsubsection{Les constructions de type $: \mathrm{V}+\mathrm{N}$}

Comme précédemment, les constituants de l'unité complexe construite appartiennent à des classes grammaticales différentes. Ici, c'est un verbe qui s'associe à un nom pour former une unité lexicale complexe comme l'indiquent en (18) les items suivants :
a. gbesì̀ + wàwı
(trainer + déchets $)$
$\rightarrow$ gbìsì̀-wàwı
telì̀ + su
(adosser + arbre)
$\rightarrow$ telì̀-su
'qui traine des déchets'
b. tíè + nyöố
gaà + nglố
(guider + AGENT)
$\rightarrow$ tíè-nyö
(accoucher + femme)
$\rightarrow$ gaà + nglö
'chaise esp'
klue + nyöö
(élever + AGENT)
$\rightarrow$ klue + nyö
'guide'
'nourrice'
'éleveur'

Il s'agit encore ici de composés exocentriques ; aucun constituant ne pouvant être interprété comme tête sémantique du mot construit. Il en est également des composés suivants :

$$
\begin{array}{llll}
\text { zizì̀ + dá } & (\text { cacher }+ \text { lieu }) & \rightarrow \text { zizì̀-da } & \text { 'cachette' } \\
\text { pu }+ \text { sa }+ \text { dá } & (\text { coucher }+ \text { PART. + lieu }) & \rightarrow \text { sëpù-da } & \text { 'couchette' } \\
\text { budò + dá } & (\text { laver }+ \text { lieu }) & \rightarrow \text { budò-da } & \text { 'endroit où l'on se lave' } \\
\text { budò + dá } & (\text { prier }+ \text { lieu }) & \rightarrow \text { budò-dá } & \text { 'endroit où l'on prie' } \\
\text { gamà + dá } & (\text { jouer }+ \text { lieu }) & \rightarrow \text { gamà-da } & \text { 'endroit où l'on joue' }
\end{array}
$$

Dans les composés en (18), les constituants de droite peuvent être analysés comme des arguments du verbe. En (18a), les constituants de droite ont une valeur sémantique d'instrument. En (18b), ils ont une valeur sémantique d'agent. Quant aux composés en (19), le constituant de droite est un nom dénotant, du point de vue sémantique, une valeur locative avec le sens précis de 'lieu, endroit'. Beaucoup de substantifs complexes sont construits sur cette base. Mais dans la langue, la manipulation de tels mots est délicate. ${ }^{10}$

${ }^{10}$ Les phrases ci-dessous mettent en exergue la manipulation délicate de telles expressions :
(4) $\mathrm{n}$ kஸ̀ zızìo $d a$ ní $\mathrm{n}$ niì mla-bòlú
1.SG COP cacher LOC CNJ 1.SG voir-ACC serpent
Je me cachais quand je vis un serpent.
(5) $\mathrm{n}$ kஸे budò da ní ̀̀ jì
1.SG COP laver LOC CNJ 2.SG venir-ACC
Je me lavais quand tu es arrivé.
(6) $\mathrm{n}$ kì bhibhìe da ní wa laà-n
1.SG COP prier LOC CNJ 3.PL appeler-ACC-1.SG.OBJ
Je priais quand ils m'ont appelé.

Dans ces différentes phrases, la valeur locative de 'dá' est inhibée. 'dá' répondrait ici à une modalité discursive avec la valeur de simultanéité, de concomitance. C'est l'emploi de la copule kò, dans le cas d'espèce, qui lui 


\subsubsection{Les constructions à trois bases lexicales}

\subsubsection{Les constructions de type $\mathbf{N}+\mathrm{N}+\mathrm{N}$}

Les items en (20) illustrent ce genre de mots construits :
a. bá + bheli + jú (père + frère + enfant $)$
b. tlikpe +
(européen + citron +
$\rightarrow$ bá-bhelì-ju
'coussin/cousine'
dògòbhi $+\mathrm{su}$ arbre)
tlikpغ-
'oranger'
c. zabh $\omega+$ wlú +
$($ coco + tête + gombo $)$
dògòbhì-su
bògbá
za-wlù-
'noix de palmes'
gbògba

Ces items soulèvent un problème important : celui de leur structure interne. Ils ont soit, une structure bipartite; soit, une structure tripartite. Un composé est dit tripartite si l'association des deux premières bases ou celle des deux dernières bases ne sont susceptibles de constituer des mots construits existants dans la langue. C'est le cas de l'item en (20c). Dans ce mot construit, ni l'association des deux premiers noms, ni celle des deux derniers ne permet de construire une unité lexicale complexe. Autrement dit, nous ne saurions tirer profit des déconstructions suivantes :

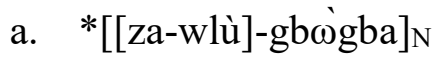
b. *[za-wlù-[gbஸ̀gba $]_{\mathrm{N}}$

Le mot désignant une 'noix de palmes' ne peut être structuré ni comme en a, ni comme en b. Il s'agit donc d'un mot construit ayant une structure tripartite.

En revanche, un composé est dit bipartite si l'association des deux premières bases ou celle des deux dernières bases est susceptible de constituer des mots construits existant dans la langue. C'est le cas des items en (20a) et (20b). Cependant, ces deux items diffèrent de par leurs structures internes sous-jacentes. Autrement dit, de la structure tripartite de surface $[\mathrm{N}-\mathrm{N}-\mathrm{N}]_{\mathrm{N}}$, deux structures internes sous-jacentes peuvent être déduites. Il s'agit des structures bipartites [[N + $\mathrm{N}]+\mathrm{N}]_{\mathrm{N}}$ et $[\mathrm{N}+[\mathrm{N}+\mathrm{N}]]_{\mathrm{N}}$. L'item en $(20 \mathrm{a})$ obéit à la première structure bipartite. Observons :
a. $[\mathrm{N}-\mathrm{N}]_{\mathrm{N}}$
bá-bheli
'oncle paternel'
b. $[[\mathrm{N}-\mathrm{N}]-\mathrm{N}]_{\mathrm{N}}$
bá-bhelì-ju
'cousin/cousine'

L'item en (20b) répond à la deuxième structure bipartite comme on peut l'observer ci-dessous :

(23) a. $[\mathrm{N}-\mathrm{N}]_{\mathrm{N}}$
dògòbhì-su
b. $[[\mathrm{N}-\mathrm{N}]-\mathrm{N}]_{\mathrm{N}}$
tlikpغ̇-dògòbhì-su 'oranger'

On pourrait représenter ces structures bipartites, respectivement, suivant les graphes ci-dessous :

\footnotetext{
suggère la valeur discursive de simultanéité ou de concomitance. D'ailleurs, ces deux unités ont un fonctionnement de morphème discontinu vis-à-vis du verbe. Ils prennent le verbe en étau.
} 

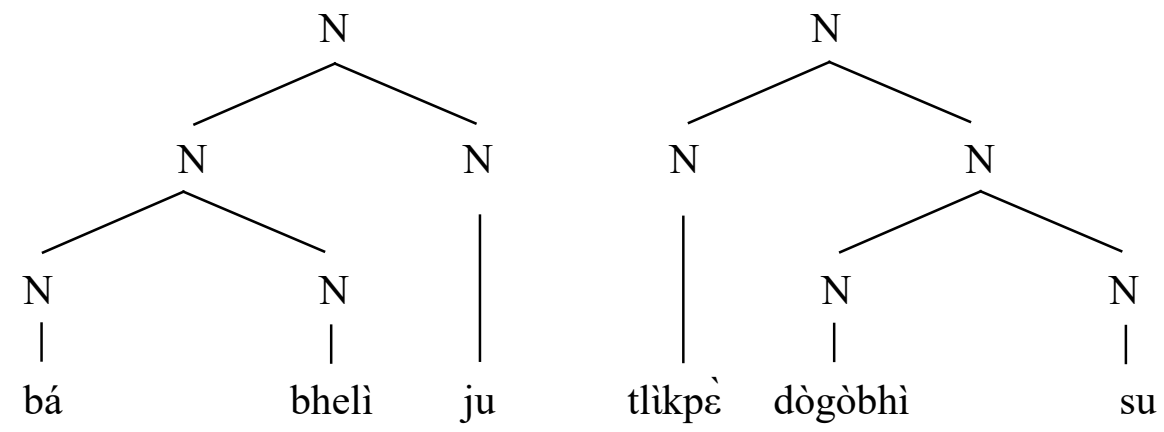

L'hypothèse des structures binaires en syntaxe et en morphologie est soutenue par la théorie linguistique défendue par Kayne (1984); Aronoff (1976); Scalise (1984); Di Sciullo (1987). Cette théorie fait appel à la notion de mot possible. ${ }^{11} \mathrm{Il}$ s'agit en fait de l'association de mots établis, non pas sur la base du mot existant, mais sur celle du mot possible ; du mot qui présenterait une structure binaire. Dans la langue, le mot bá-bhelì-ju 'coussin/cousine' est une construction candidate à la notion de mot possible. En dehors de la structure interne sous-jacente $[[\mathrm{N}-\mathrm{N}]-\mathrm{N}]_{\mathrm{N}}$ qu'on lui a reconnu, ce mot peut admettre la structure binaire $[\mathrm{N}[\mathrm{N}-\mathrm{N}]]_{\mathrm{N}}$ : [bá[bhelì-ju] $]_{N}$. Dans cette structure, le segment [bhelì-ju] est une séquence de mot possible :

$$
\begin{aligned}
& \text { bhelì-ju } \\
& \text { frère-enfant }
\end{aligned}
$$

Il s'agit d'un mot que tout locuteur godié peut accepter, admettre, mais qui ne saurait faire l'objet d'une entrée de dictionnaire. En revanche, le mot construit tlikpè-dògòbhi-su 'oranger' admet les deux structures binaires $[[\mathrm{N}-\mathrm{N}] \mathrm{N}]_{\mathrm{N}}$ et $[\mathrm{N}[\mathrm{N}-\mathrm{N}]]_{\mathrm{N}}$. En effet, les segments [tlikpغ́dògòbhi] et [dògòbhì-su] peuvent faire l'objet d'entrées de dictionnaire. Il s'agit de deux mots bien formés dans la langue. tlikpé-dògòbhi signifie 'orange ou citron de blanc' ; dògòbhi-su signifie 'citronnier'.

\subsubsection{Les constructions de type $\mathrm{N}+\mathrm{V}+\mathrm{N}$}

Les items ci-dessous illustrent ce type de mots construits : ${ }^{12}$

$$
\begin{aligned}
& \text { jlá }+ \text { kốs'́ }+\quad \text { (demander }+ \text { commerce }+\quad \rightarrow \text { kốs'́-jlà- 'commerçant' } \\
& \text { nyöö́ AGENT) } \\
& \text { nyö } \\
& \text { plá }+ \text { klá }+\quad(\text { entrer + champ }+ \text { AGENT }) \quad \rightarrow \text { klá-plà- } \quad \text { 'chasseur' } \\
& \text { nyöố } \\
& \text { nyö }
\end{aligned}
$$

\footnotetext{
${ }^{11}$ De nombreux auteurs font état de la dichotomie mot possible/mot existant. On considère qu'un mot est qualifié de «possible » s'il peut être accepté par les locuteurs d'une langue comme bien formé sans faire pour cela, l'objet d'une entrée de dictionnaire. Le mot existant, par contre, c'est celui que tout locuteur d'une langue reconnait comme bien formé et pouvant faire l'objet d'une entrée de dictionnaire (cf. Corbin 1987, 1997 ; Dressler/Ladányi 2000 ; Plag 1999 ; Bauer 2001). Mais certains comme Dal (1997, 2003) considère qu'on ne peut opposer mots possibles et mots existants, car « les mots construits existants sont un sous-ensemble des mots possibles ».

${ }^{12}$ Dans ces exemples, les deux constituants les plus à gauche forment ensemble une base verbale complexe. Ainsi : jlá + kốsé signifie 'faire le commerce ou commercer';

plá + klá signifie 'faire la chasse ou chasser';

$n \omega+$ lebhe signifie 'travailler';

$l i+a ̀ J a$ signifie 'hériter'.
}

Alors, les constituants nominaux kö́sé 'commerce', klá 'champ', lebhe 'travail' et à $J a$ 'héritage' doivent être considérés ici comme des extensions du verbe et non comme des compléments. 


$$
\begin{aligned}
& \mathrm{n} \omega+\text { lebhe } \quad(\text { faire + travail }+ \text { AGENT) } \rightarrow \text { lebhè-n } \omega \text { - 'travailleur' } \\
& + \text { nyöó nyö } \\
& \mathrm{li}+\text { àJa }+ \text { (manger + héritage + AGENT } \rightarrow \text { àJa-lì-nyö 'héritier' } \\
& \text { nyöö }
\end{aligned}
$$

Comme nous pouvons le constater, les mots construits ci-dessus sont constitués de deux substantifs encadrant un lexème verbal. Le lexème verbal et le substantif le plus à gauche forment une base verbale complexe. La structure segmentale profonde de cette base verbale pourrait être configurée comme suit : [VN]v. Les verbes qui ont cette structure sont tous des locutions verbales. Ils ont un fonctionnement syntaxique de morphèmes discontinus ${ }^{13}$. Dans ce type de bases verbales complexes, en effet, les lexèmes verbaux et les substantifs qui leurs sont associés fonctionnent comme des particules séparables, à l'instar de certains verbes allemands. Soit le verbe n $\omega$ lebhe 'travailler'. Il est composé du verbe n $\omega$ 'faire' et de lebhe 'travail'. On peut observer le fonctionnement discontinu de ce verbe dans l'énoncé suivant :

$$
\begin{array}{lllllllll}
\text { dàgố } & \text { n } & \text { [zeplí } & \text { ó } & \text { wótồ-nglö } & \text { á } & \text { klá } & \text { mlí } & \underline{\text { lebhe }} \\
\text { n.p } & \text { faire- } & \text { [matin } & \text { 1.POSS } & \text { belle-mère } & \text { CON } & \text { champ } & \text { POST } & \text { travail } \\
& \text { INACC } & & & & & &
\end{array}
$$

Dago travaille ce matin dans la plantation de sa belle-mère.

Nous le voyons, les unités soulignées forment une base verbale complexe. Elles ont effectivement un fonctionnement discontinu et traitées, à ce titre, comme des particules séparables. Considérant le segment entre crochet comme une expansion non indispensable, l'énoncé peut être réduit comme suit :
dàgố $\underline{n \omega}$
lebhe
n.p faire-INACC travail
Dago travaille.
Litt. : 'Dago fait travail.'

Sous le contrôle de la relation prédicat-argument, nous pourrions attribuer au constituant nominal lebhe 'travail' la fonction grammaticale d'objet. D'ailleurs, la structure syntaxique même de l'énoncé, ainsi que sa traduction littérale en français, l'indiquent bien. De cet énoncé, nous pouvons déduire celui-ci :
dàgố plì
lebhè-n⿳亠丷-nyö
n.p COP
travail-faire-AGENT
Dago est un travailleur.

A Dago, on attribue ainsi, du point de vue pragmatique, toutes les qualités d'un homme vaillant, courageux, travailleur et même serviable.

\footnotetext{
${ }^{13}$ La caractérisation des bases verbales complexes comme ayant dans la langue un fonctionnement de morphèmes discontinus se justifie dans l'exemple (26) avec la portion de phrase mis entre crochets. Dans cet exemple, le constituant nominal lebhe 'travail' n'est pas en position objectale, loin s'en faut. Il s'agit ici d'un constituant nominal en position d'extension. C'est-à-dire que la partie nominale de la base verbale complexe se détache du radical verbal avec l'insertion, dans la phrase, d'un groupe de mots en position d'expansion. La suppression d'un tel groupe de mots n'entache en rien le sens global de l'énoncé. Il ne s'agit donc pas, comme on peut le croire, d'un objet du verbe.
} 
Nous le voyons, dans la construction de mots nouveaux avec les bases verbales complexes, le verbe, noyau du lexème verbal composé, permute avec sa partie nominale, occupant ainsi la position médiane. Ici, le segment [-nyö] - qui substitue le terme sujet - a une valeur sémantique d'agent. C'est ce qui explique la glose AGENT que nous lui attribuons. D'ailleurs, c'est cette valeur qu'on attribue généralement aux mots construits à partir de bases verbales complexes. Ces mots construits sont généralement traités de « composés synthétiques » (cf. Cahill 2007, cité par Anderson 2013 : 93).

Les mots construits ayant la structure $[\mathrm{N}-\mathrm{V}-\mathrm{N}]_{\mathrm{N}}$ sont généralement qualifiés de composés synthétiques. On considère que ce type de composés emprunte à la fois à la dérivation et à la composition. Quand ils obéissent au procédé de dérivation, ils correspondraient à la structure sousjacente [[N-V]-nyö]N. Quand, c'est au procédé de composition qu'ils obéissent, ils répondraient à la structure sous-jacente $[\mathrm{N}-[\mathrm{V}-\mathrm{nyö}]]_{\mathrm{N}}$. Ces deux approches de mots construits sont défendues, respectivement, par Lieber (1981) et par Selkirk (1982). Elles sont bien applicables aux mots construits répondant à la structure tripartite de surface $[\mathrm{N}-\mathrm{V}-\mathrm{N}]_{\mathrm{N}}$. Dans la langue, la structure sous-jacente $[[\mathrm{N}-\mathrm{V}]-\text { nyö }]_{\mathrm{N}}$ sied aux mots construits à partir de bases verbales complexes. ${ }^{14}$ Quant à la structure $\left[\mathrm{N}-[\mathrm{V} \text {-nyö] }]_{\mathrm{N}}\right.$, elle sied aux mots construits à partir de verbes à objets inhérents. Dans la structure interne sous-jacente $[[\mathrm{N}-\mathrm{V}]-\text { nyö }]_{\mathrm{N}}$, le constituant nominal le plus à droite, c'est-à-dire -nyö, agit comme un modifieur syntagmatique ; le segment [NV] de la structure $[[\mathrm{N}-\mathrm{V}]-n y o ̈]_{\mathrm{N}}$ étant déduite de la relation prédicat-argument. Par contre, ce n'est pas le cas du segment $\left[\mathrm{V}\right.$-nyö] de la structure interne sous-jacente $[\mathrm{N}-[\mathrm{V}-\mathrm{nyö}]]_{\mathrm{N}}$. Dans le segment $[\mathrm{V}$ nyö], en effet, le constituant -nyö modifie le verbe en lui attribuant des valeurs grammaticales de nom. C'est ce qu'illustrent les items en (18a) et en (18b). Dans la structure sous-jacente [N[V-nyö] $]_{N}$, le constituant $\mathrm{N}$ est un objet inhérent au verbe. Il complexifie le segment [V-nyö] en lui apportant beaucoup plus de précision dans sa définition. Les exemples ci-dessous en sont une illustration :

\begin{tabular}{|c|c|c|c|c|}
\hline tíè + göl & $\begin{array}{l}\text { (conduire }+ \text { pirogue } \\
+ \text { AGENT) }\end{array}$ & $\rightarrow$ & 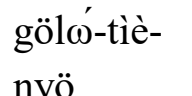 & 'piroguier' \\
\hline tíè + tomobí + nyöô & $\begin{array}{l}\text { (conduire + automo- } \\
\text { bile }+ \text { AGENT) }\end{array}$ & $\rightarrow$ & $\begin{array}{l}\text { tomobíì- } \\
\text { tìè-nyö }\end{array}$ & $\begin{array}{l}\text { 'conducteur } \\
\text { d'automobile, } \\
\text { chauffeur' }\end{array}$ \\
\hline klá + golù + nyöö & $\begin{array}{l}(\text { champ }+ \text { cultiver }+ \\
\text { AGENT })\end{array}$ & $\rightarrow$ & $\begin{array}{l}\text { klá-gòlù- } \\
\text { nyö }\end{array}$ & $\begin{array}{l}\text { 'cultivateur/agri- } \\
\text { culteur' }\end{array}$ \\
\hline loku + bhla + nyöó & $\begin{array}{l}\text { (pagnes }+ \text { tisser }+ \\
\text { AGENT) }\end{array}$ & $\rightarrow$ & $\begin{array}{l}\text { lokù-bhlà- } \\
\text { nyö }\end{array}$ & 'tissérand' \\
\hline loku + kplá + nyöố & $\begin{array}{l}\text { (pagnes }+ \text { coudre }+ \\
\text { AGENT) }\end{array}$ & $\rightarrow$ & $\begin{array}{l}\text { lokù-kplà- } \\
\text { nyö }\end{array}$ & 'couturier' \\
\hline bhùtu + slá + nyöố & $\begin{array}{l}\text { (maison }+ \text { construire } \\
+ \text { AGENT) }\end{array}$ & $\rightarrow$ & $\begin{array}{l}\text { bhùtù-slà- } \\
\text { nyö }\end{array}$ & 'maçon' \\
\hline$\dot{o}+$ bhlı + nyöố & $\begin{array}{l}\text { (chanson }+ \text { chanter }+ \\
\text { AGENT) }\end{array}$ & $\rightarrow$ & $\begin{array}{l}\text { la-bhlì- } \\
\text { nyö }\end{array}$ & 'chanteur' \\
\hline
\end{tabular}

\footnotetext{
${ }^{14}$ Nous voulons rappeler ici que, dans le procédé de construction de tels mots, la base verbale et sa partie nominale permutent. Ce qui permet au verbe de recevoir le constituant nominal qui s'associe pour construire le mot nouveau.
} 


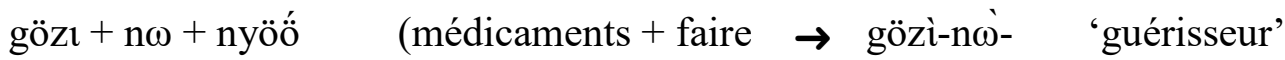

$$
\begin{aligned}
& + \text { AGENT) nyö } \\
& \text { gbàlí }+\mathrm{z} \varepsilon+\text { nglố } \quad \text { (buches }+ \text { couper }+\quad \rightarrow \text { gbàlí-zغं- 'bucheronne' } \\
& \text { femme) nglö } \\
& \text { gblùú }+ \text { lozò + nyöố } \quad \text { (forge }+ \text { traiter }+\quad \rightarrow \text { gblu-lòzò- 'forgeron' } \\
& \text { AGENT) nyö }
\end{aligned}
$$

Il s'agit en réalité de verbes transitifs dont la réalisation de l'objet direct n'est pas facultative ; « celui-ci [étant] responsable d'une partie de la signification » de telle constructions de mots. ${ }^{15}$ Dans ces exemples en (29), les constituants -nyö et -nglö ont une valeur agentive. Ils s'accolent au verbe pour former un nom [V-nyö $]_{\mathrm{N}}$ ou $[\mathrm{V} \text {-nglö }]_{\mathrm{N}}$. Ainsi, dans la structure $\left[\mathrm{N}-[\mathrm{V} \text {-nyö] }]_{\mathrm{N}}\right.$, le constituant $\mathrm{N}$ fonctionne comme un argument interne. Le constituant $\mathrm{N}$ de la structure $[[\mathrm{N}-\mathrm{V}]-$ nyö $]_{N}$ fonctionne également, de façon apparente, comme un argument interne. Du moins, on pourrait lui reconnaitre cette valeur; bien qu'il soit une partie constitutive d'une base verbale complexe. Ainsi, la structure tripartite de surface $[\mathrm{N}-\mathrm{V}-\mathrm{N}]_{\mathrm{N}}$ répond à deux structures binaires sous-jacentes que sont les structures $[[\mathrm{N}-\mathrm{V}]-n y o ̈]_{\mathrm{N}}$ et $[\mathrm{N}-[\mathrm{V}-\mathrm{nyö}]]_{\mathrm{N}}$. Leurs représentations arborescentes respectives, donnent les graphes suivants :

\begin{tabular}{|c|c|c|c|c|c|c|c|}
\hline lebhè & $\underline{\mathrm{n \omega}}$ & nyö & 'travailleur' & göló & $\underline{\text { tì̀ }}$ & nyö & 'piroguier' \\
\hline àJa & lì & nyö & 'héritier' & la & bhlì & nyö & 'chanteur' \\
\hline & plà & nyö & 'chasseur' & gblu & lòzò & nyö & 'forgeron' \\
\hline
\end{tabular}

(30a)

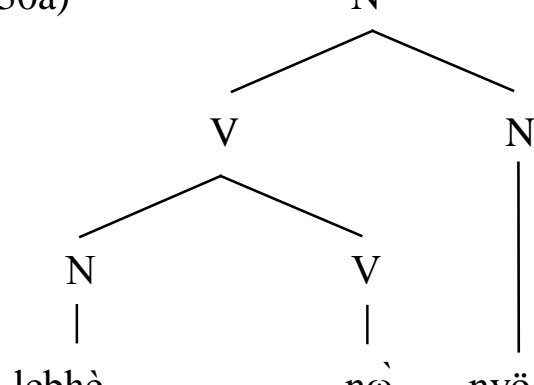

(30b)

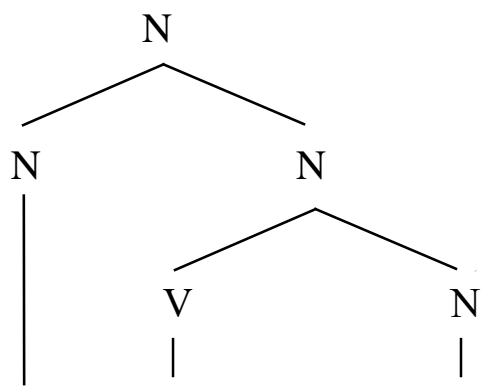

NB : La structure tripartite $[\mathrm{N}-\mathrm{V}-\mathrm{N}]_{\mathrm{N}}$ existe aussi pour une autre classe de mots construits. Il s'agit de mots construits dont la déconstruction en l'une ou l'autre des deux structures ci-dessus énumérées est impossible. C'est le cas des exemples suivants :

$$
\begin{aligned}
& \text { bhlu + sáká }+ \text { gòdë (piler + riz + mortier) } \rightarrow \text { sáká-bhlù- (mortier pour } \\
& \text { gòdë riz) } \\
& \text { bhlu }+ \text { gui }+ \text { gòdë } \quad \text { (piler }+ \text { graine de } \quad \rightarrow \text { gui-bhlù- } \quad \text { (mortier pour } \\
& \text { palme }+ \text { mortier } \quad \text { gòdë graines de } \\
& \text { palmes) }
\end{aligned}
$$

Dans ces exemples, le constituant le plus à droite - gòdë 'mortier' - a une valeur sémantique d'instrument; et le constituant le plus à gauche, qui semble fonctionner comme l'argument interne, a la valeur de qualifiant ou de spécificateur. ${ }^{16}$

\footnotetext{
15 On pourrait penser que, dans les exemples en (29), l'objet direct est facultatif. Il n'en est rien en réalité. L'item tîè-nyö des exemples en (18b) donne cette impression. Il signifie à vrai dire 'guide, éclaireur'. Pour signifier 'conducteur ou chauffeur', l'on est obliger d'adjoindre à ce mot composé un objet direct, en l'occurrence le terme tomobí 'autmobile', terme emprunté au français : tomobî̀-tìe-nyö. En l'espèce, l'objet direct ne saurait être facultatif.

16 Nous pensons que le statut de «mots construits » attribué à ces constructions peut être discuté.
} 


\subsection{Les séquences syntaxiques de type $\mathrm{N}+\mathrm{ADV}+\mathrm{N}^{17}$}

Illustrent ce type de mots construits, les deux items suivants :

$$
\begin{aligned}
& \text { blï + zồ.n + walı (cou+sous.LOC+affaire }) \rightarrow \text { blı-zồ.n-wàlı 'voix' } \\
& \text { klá }+ \text { zồ.n }+ \text { bhli (bois+sous.LOC+bœuf) } \rightarrow \text { klá-zồ.n-bhlií 'biche noire' }
\end{aligned}
$$

Dans ces items, chaque séquence syntaxique est la somme de deux substantifs encadrant une postposition. Les constituants les plus à gauche de chaque séquence forment un syntagme adverbial. Les exemples ci-dessous donnent une illustration :
a. blı zồ.n
cou en bas.LOC
En bas du cou.
b. klá zồ.n
En bas des champs.
champ en bas.LOC

Le syntagme adverbial blı zö.n, dont la traduction littérale donne 'en bas du cou', signifie 'gorge' dans la langue. On peut le constater dans l'énoncé suivant :
(34) ná
bli-zö̀n
bhlaá-n
1.POSS cou-sous.LOC
tuer-INACC.1SG.OBJ

Ma gorge me fait mal.

Litt. : « Ma gorge me tue ».

Ce syntagme adverbial semble fonctionner comme un mot construit. Sa traduction en français suggère cette appréciation. Ce qui n'est nullement le cas de la séquence klá zö̀.n qui reste toujours un syntagme adverbial. Il signifie 'les sous bois', comme l'atteste l'énoncé ci-dessous :
$\mathrm{n}$ $\mathrm{m} \omega$ klá champ
zồ.n
1.SG aller-INACC
Je vais dans les sous bois.

Ainsi, bien qu'ayant, en apparence, la même structure syntaxique [N-ADV $]_{\mathrm{SADV}}$, les deux constructions suggèrent deux catégories grammaticales différentes. L'un a toutes les caractéristiques d'un mot construit; l'autre reste un groupe syntaxique. La même suggestion semble plausible pour les deux items en (32), respectivement. Le premier est un nom; le second un syntagme. Les deux énoncés respectifs en donnent une illustration :
(36) jú-á

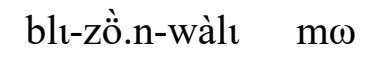
títí
enfant-DEF voix
aller-HAB
ADV
L'enfant a une voix qui porte vraiment.
(37) zeplí
dàgố
bhlaà
matin
n.p.
tuer-ACC
klá-zồ.n-bhlìi
biche noire.

Ce matin, Dago a tué une biche noire.

\footnotetext{
${ }^{17}$ La valeur adverbiale que nous accordons au terme zồ vient du fait qu'il soit associé à un locatif. En principe, il s'agit d'une postposition.
} 
Litt. : Dago a tué un bœuf des sous bois.

Mais, que l'un soit interprété comme un mot construit et l'autre comme un groupe syntaxique, il faut admettre qu'il s'agit bel et bien de séquences syntaxiques lexicalisées. Leur structure tripartite de surface $[\mathrm{N}-\mathrm{ADV}-\mathrm{N}]_{\mathrm{N}}$, répond à la structure binaire sous-jacente $[[\mathrm{N}-\mathrm{ADV}]-\mathrm{N}]_{\mathrm{N}}$. Sur la base de cette structure sous-jacente, on pourra avoir pour ces deux items la représentation arborescente ci-dessous :

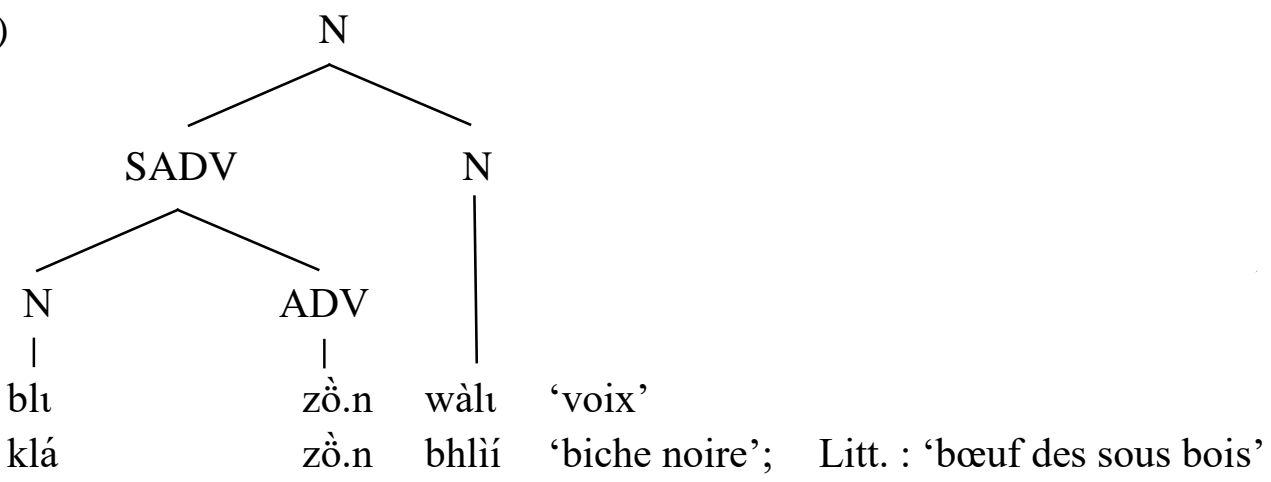

\subsection{Les séquences syntaxiques de type $\mathrm{N}+\mathrm{A}+\mathrm{V}+\mathrm{N}$}

Les items suivants permettent d'illustrer ce type de mots construits :

$$
\begin{array}{llll}
\text { bhlá+mla+glui+cıć́ } & \begin{array}{l}
\text { (tuer+ani- } \\
\text { maux+gros+aigle) } \\
\text { bhlá+gu+zàl } \omega+\text { +nyöố }
\end{array} & \begin{array}{l}
\text { (tuer+mala- } \\
\text { die+rouge+AGENT) }
\end{array} & \rightarrow \text { gu-zàl } \omega \text {-bhlà-nyö 'lépreux' }
\end{array}
$$

Les séquences syntaxiques lexicalisées ci-dessus sont constituées de deux substantifs encadrant un adjectif et un verbe. Dans chaque séquence, les deux constituants les plus à gauche constituent déjà dans la langue un mot construit, formé d'un nom et d'un adjectif. Observons :

$$
\begin{array}{llllll}
{[\mathrm{N}-\mathrm{A}]_{\mathrm{SA}}} & & & & \\
\text { mla }+ \text { glui } & \text { (animaux }+ \text { gros) } & \rightarrow & \text { mla-glùi } & \text { 'gros animaux' } & \\
\text { gu }+ \text { zàl } \omega & \text { (maladie }+ \text { rouge) } & \rightarrow & \text { gu-zàl } \omega & \text { 'lèpre' } & \text { lit. «maladie }
\end{array}
$$$$
\text { rouge » }
$$

Il s'agit en réalité, pour ces deux items, de syntagmes adjectivaux. La signification du premier est déductible de ces composantes. Celle du second l'est également, si l'on se réfère à la traduction littérale, mais complètement imprévisible, si l'on s'en tient à son sens exact en français. Les séquences syntaxiques lexicalisées de structure $[\mathrm{N}-\mathrm{A}-\mathrm{V}-\mathrm{N}]_{\mathrm{N}}$ pourraient être qualifiées de « constructions synthétiques », par analogie aux composés synthétiques. Mais à la différence des mots construits $[\mathrm{N}-\mathrm{V}-\mathrm{N}]_{\mathrm{N}}$, qui requièrent deux structures sous-jacentes (cf. supra), une seule structure sous-jacente est retenue pour les séquences de structure $[\mathrm{N}-\mathrm{A}-\mathrm{V}-\mathrm{N}]_{\mathrm{N}}$. Il s'agit de la structure sous-jacente $\left[[\mathrm{N}-\mathrm{A}]_{\mathrm{N}}-\mathrm{V}-\mathrm{N}\right]_{\mathrm{N}}$. Sur la base de cette structure, on pourrait donner, pour ces deux items, la représentation graphique suivante : 
(41)

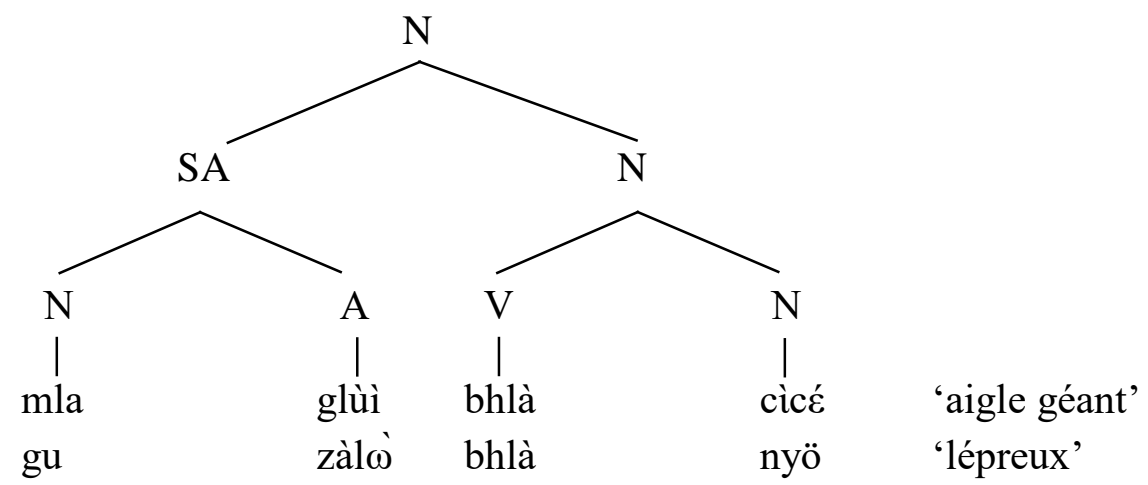

En instituant sur la notion du mot possible, pour le segment [V-N], on pourrait considérer la structure quadripartite $[\mathrm{N}-\mathrm{A}-\mathrm{V}-\mathrm{N}]_{\mathrm{N}}$ comme une structure de surface de la structure bipartite sous-jacente $[[\mathrm{N}-\mathrm{A}]-\mathrm{V}-\mathrm{N}]_{\mathrm{N}}$. Autrement dit, il s'agit, en l'espèce, de deux mots construits qui s'associent pour donner naissance à des séquences syntaxiques lexicalisées : $\left[[\mathrm{N}-\mathrm{A}]_{\mathrm{SA}}+[\mathrm{V}-\right.$ $\left.\mathrm{N}]_{\mathrm{N}}\right] \rightarrow[[\mathrm{N}-\mathrm{A}]-\mathrm{V}-\mathrm{N}]_{\mathrm{N}}$. Les deux séquences ci-dessus peuvent être glosées respectivement comme suit :

(42) a. 'L'espèce d'aigle qui tue de gros animaux'.

b. 'L'homme qui est atteint de la lèpre'.

Il s'agit bien évidemment, d'expressions syntaxiques lexicalisées. Ainsi, sommes-nous d'avis avec Fabb (1984) et Hoeksema (1985) que, tout composé contenant un syntagme est analysé comme ayant une structure partiellement syntaxique. Cela est d'autant plus vrai que l'énoncé suivant :

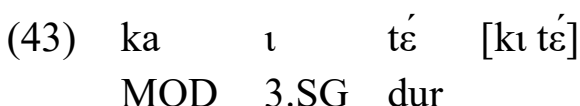

C'est dur/c'est difficile.

Permet d'obtenir la séquence syntaxique lexicalisée ci-dessous :

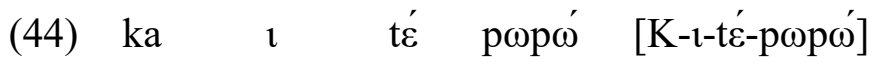

MOD 3.SG dur monde

Un monde difficile.

Lequel permet de construire l'énoncé suivant :

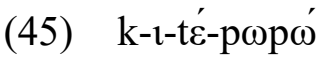

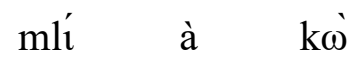
MOD-3.SG-dur-monde POST 1.PL COP
Nous sommes dans un monde difficile.

\subsection{Les séquences syntaxiques de type $\mathrm{N}+\mathrm{POST}+\mathrm{V}+\mathrm{N}$}

Les séquences syntaxiques de structure $[\mathrm{N}-\mathrm{POST}-\mathrm{V}-\mathrm{N}]_{\mathrm{N}}$ sont illustrés, dans les limites de notre corpus, par l'item suivant :

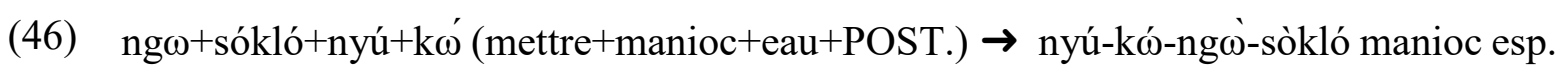

Dans cet exemple, l'approche syntaxique de l'unité lexicale complexe est encore plus plausible. En effet, la structure syntaxique sous-jacente, de laquelle est déduite le mot construit ci-dessus, 
répond dans l'absolue, à un ordre adressé à un récepteur. Il s'agit d'une construction à l'impératif :

$\begin{array}{llll}\text { ng } \omega & \text { sókló } & \text { nyú } & \mathrm{k} \omega \\ \text { mettre-IMP } & \text { manioc } & \text { eau } & \text { POST }\end{array}$

Mets le manioc dans l'eau.

Cet état de fait a permis d'attribuer à cette espèce de tubercule la dénomination de $n y u ́-k \omega ́-\eta w \omega$ sòkló, littéralement glosée en français comme suit :

'Du manioc mis dans de l'eau'.

C'est une espèce de tubercule de manioc qui ne peut être consommée qu'après être mise dans de l'eau pendant un ou deux jours.

De la structure $[\mathrm{N}-\mathrm{POST}-\mathrm{V}-\mathrm{N}]_{\mathrm{N}}$, on ne saurait tirer profit des arguments en faveur de la bipartition structurelle interne des composants comme il a été question dans les items en (39). Autrement dit, la décomposition de la structure $[\mathrm{N}-\mathrm{POST}-\mathrm{V}-\mathrm{N}]_{\mathrm{N}}$ en structure sous-jacente virtuelle $[[\mathrm{N}-\mathrm{POST}]-\mathrm{V}-\mathrm{N}]_{\mathrm{N}}$ ou $[\mathrm{N}-\mathrm{POST}-[\mathrm{V}-\mathrm{N}]]_{\mathrm{N}}$ est inopérante.

\subsection{Les séquences syntaxiques de type $\mathrm{N}+\mathrm{ADV}+\mathrm{N}+\mathrm{N}$}

Les séquences syntaxiques de structure $[\mathrm{N}-\mathrm{ADV}-\mathrm{N}-\mathrm{N}]_{\mathrm{N}}$ sont illustrés, également, dans les limites de notre corpus, par un seul item. Il s'agit de l'item :

$$
\begin{aligned}
& \text { blï+zồ.n+nglố (cou+sous.LOC+ } \rightarrow \text { bli-zồ.n-nglö̀- 'aluette/pomme } \\
& \text { +gbojo femme+bourgeon) gbàjo d'Adam' }
\end{aligned}
$$

La structure de cette séquence est $[\mathrm{N}-\mathrm{ADV}-\mathrm{N}-\mathrm{N}]_{\mathrm{N}}$. Contrairement à l'item en (46), ici, on peut tirer profit des arguments en faveur de la bipartition de la structure interne des composants. Il s'agit d'une structure de surface issue de la structure bipartite sous-jacente [[N-ADV] $]_{\text {SADV-N- }}$ $\mathrm{N}]_{\mathrm{N}}$; le segment $[\mathrm{N}-\mathrm{ADV}]_{\mathrm{SADV}}$ ayant été déjà analysé en filigrane comme un mot construit dans la langue suivant la construction en (33a) que nous reprenons ici en (50) :

$$
\begin{aligned}
& {[\mathrm{N}-\mathrm{ADV}]_{\mathrm{SADV}}} \\
& \text { blì }+ \text { zò̀.n }
\end{aligned}
$$

Ce syntagme adverbial donne lieu à un mot construit dans la langue, comme nous l'avons déjà souligné. C'est son association à la composante nglö-gbàjo qui aboutit à la séquence syntaxique lexicalisé bll-zö̀.n-nglö-gbàjo signifiant 'alouette ou pomme d'Adam'. Il est entendu que la composante nglö-gbàjo, dérivée de nglö 'femme' et de gbojo 'bourgeon', n'a aucune valeur sémantique, prise isolement. Mais son association à la composante bll-zö̀.n 'gorge' donne lieu à la construction syntaxique lexicalisée : bll-zö.n-nglö-gbàjo « alouette ou pomme d'Adam ».

\section{Conclusion}

Au total, on retiendra que les mécanismes de construction de mots sont complexes. La dérivation met en relief deux dérivatifs de substantivation de verbes ( $-l l$ et $-n o ̈)$ et un dérivatif à valeur

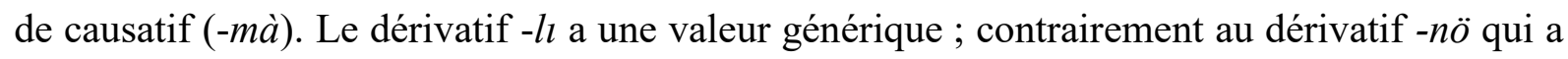
une valeur spécifique. Il ne permet de substantiver que des verbes de caractérisation. Il n'existe pas, dans la langue, un procédé de construction de verbes à partir de nom. La composition met 
en relief deux procédés de construction de mots nouveaux. Dans les procédés les plus simples, sont associées, soit deux noms ; soit un nom est adjectif; soit enfin un nom et un verbe. Dans les procédés les plus complexes, sont associés soit trois noms; soit deux noms et un verbe. Dans les séquences syntaxiques lexicalisées, on a analysé des séquences de trois ou de quatre composantes. Il s'agit d'unités linguistiques complexes très peu répandues. Seulement un ou deux exemples ont permis d'illustrer chaque cas. Entre ces unités linguistiques complexes et les mots construits par association de deux ou de trois unités lexicales, la limite est floue. D'abord, la plupart des mots construits répond à la structure du syntagme génitival ou à celle du syntagme épithétique. Ces deux types de structures ressortent de la syntaxe. Ensuite, le critère de l'inséparabilité des constituants dans le mot construit est applicable aux séquences syntaxiques lexicalisées. D'ailleurs, dans la langue, les séquences syntaxiques lexicalisées peuvent être des entrées de dictionnaire. De plus, certaines séquences lexicalisées acceptent, comme d'autres mots construits, l'une ou l'autre des deux structures binaires sous-jacente, déduite de la structure tripartite de surface.

\section{Bibliographie}

Anderson, Jonathan (2013): "Verb-internal compound formation in akan". The Journal of west African languages 40/1: 89-104.

Anderson, Stephen (1992): A-morphous Morphology, Cambridge: Cambridge University.

Aronoff, Marc (1976): Word Formation in Generative Grammar. Cambridge: MIT.

Bassac, Christian (2003) : «Sur quelques aspects de la composition nominale en turc : le point de vue du Lexique Génératif ». Morphologie et Lexique. Cahiers de Grammaire 28 : $197-$ 215.

Bisetto, Antonietta/Scalise, Sergio (2005): "Selection in compounding and derivation". In: Dressler, Wolfgang et al. (eds.): Morphology and Its Demarcations. Amsterdam/Philadelphia, John Benjamins: 113-150.

Bauer, Laurie (2001): Morphological Productivity. Cambridge: Cambridge University.

Booij, Geert (1977): Dutch Morphology. A Study of Word Formation in Generative Grammar. Dordrecht: Foris.

Booij, Geert (2005): The Grammar of Words: An Introduction to Linguistic Morphology. Oxford: Oxford University.

Booij, Geert (2007): “Construction Morphology and the lexicon”. In: Montermini, Fabio/Boyé, Gilles/Hathout, Nabil (eds.): Selected proceedings of the $5^{\text {th }}$ Décembrettes: Morphology in Toulouse. Somerville, MA: Cascadilla Proceeding Project.

Booij, Geert (2009) : « La morphologie constructionnelle - un aperçu ». Memoires de la Société de Linguistique de Paris 17 : 13-32.

Brousseau, Anne-Marie (1989) : «Les noms composés en haitien : pour une définition intrinsèque de tête morphologique ». Revue québécoise de linguistique 18/2 : 11-39.

Brousseau, Anne-Marie (1990) : « Panorama de morphologie du fongbé ». Journal of West Africa Languages 20/1 : 27-44.

Cahill, Michael (2007): Aspects of the morphology and phonology of kDnni. Dallas: SIL International.

Corbin, Danielle (1987): Morphologie dérivationnelle et structuration du lexique. Tübingen: Niemeyer. 
Corbin, Danielle (1992) : « Hypothèses sur les frontières de la composition nominale », Cahiers de grammaire $17: 25-55$.

Corbin, Danielle (1997) : «Entre les mots possibles et les mots existants : les unités lexicales à faible probabilité d'actualisation ». In : Corbin, Danielle et al. (eds.) : Silexicales. Mots possibles et mots existants. Lille, Centre de Gestion de l'Edition Scientifique : 79-90.

Dal, Georgette (1997a) : «Tous les mots existants sont-ils possibles? ». In : Corbin, Danielle et al. (eds.) : Silexicales. Mots possibles et mots existants. Lille, Centre de Gestion de l'Edition Scientifique : 91-100.

Dal, Georgette (1997b) : « Du principe d'unicité catégorielle au principe d'unicité sémantique : incidence sur la formalisation du lexique construit morphologiquement ». Bulag numéro spécial : 105-115.

Dal, Georgette (2003): «Productivité morphologique : définitions et notions connexes ». Langue française $140: 3-23$.

Di Sciullo, Anna-Maria/Williams, Edwin (1987): On the definition of Word. Cambridge: MIT.

Dressler, Wolfgang/Ladányi, Maria (2000): "Productivity in Word Formation (WF): A Morphological Approach”. Ada Linguistica Hungarica 47/2: 103-144.

Fabb, Nigel (1984): Syntactic Affixation. PhD Dissertation, Cambridge: MIT.

Fabb, Nigel (2001): Compounding. In: Spencer, Andrew/Zwicky, Arnold (eds.): The handbook of morphology. Oxford: Blackwell.

Godé, Victor (2008) : Le dadjriwalé, langue kru de la Côte d'Ivoire. Paris : Harmattan.

Gross, Maurice (1981) : «Les bases empiriques de la notion de prédicat sémantique ». Langages $63: 8-52$.

Haspelmath, Martin (2002): Understanding Morphology. London, Arnold.

Hoeksema, Jack (1985): Categorial Morphology. New York: Garland.

Kayne, Richard (1984): Connectedness and Binary Branching. Dordrecht: Foris.

Kipré Blé, François et al. (2013) : Orthographe harmonisee des langues kru (de Cote d'Ivoire et $d u$ Liberia). Cape Town : CASAS.

Lehmann, Christian (1980): "Guidelines for interlinear morphemic translation". Institut für Sprachwissenschaft - Universität Köln, Arbeitspapier 37: 1-23.

Lieber, Rochelle (1992a): Deconstruction Morphology: Word Formation in Syntactic Theory. Chicago: University of Chicago.

Lieber, Rochelle (1992b): “Compounding in English”. Rivista di Linguistica 4/1: 79-96.

Marchese, Lynell (1983) : Atlas linguistique kru. Abidjan: Institut de Linguistique Appliquée (ILA).

Marchese, Lynell (1989): “Kru”. In: Bendor-Samuel, John (ed.): The Niger-Congo languages. Lanham, University Press of America: 119-139.

Matthews, Peters (1974): Morphology. Cambridge: Cambridge University.

N'dré, Damanan (2011): «Les constructions causatives en dadjriwalé ». Revue Ivoirienne des Sciences du langage et de la communication 5 : 197-209.

N'dré, Damanan (2012) : «Les constructions passives, réfléchies et réciproques en dadjriwalé ». Revue scientifique des arts, de la culture, des lettres et sciences humaines 3/1 : 213241.

Payne, Thomas (1997): Describing morphosyntax: a guide for field linguists. Cambridge: Cambridge University. 
Plag, Ingo (1999): Morphological Productivity. Structural Constraints in English Derivation. Berlin/New York: Mouton de Gruyter.

Plag, Ingo (2003): Word Formation in English. Cambridge: Cambridge University.

Scalise, Sergio (1984): Generative Morphology. Dordrecht: Foris.

Scalise, Sergio/Guevara, Emiliano Raul (2006): "Exocentric compounding in a typological framework". Lingue e linguaggio 2: 185-206.

Selkirk, Elisabeth (1982): The Syntax of Words. Cambridge: MIT.

Spencer, Andrew (1991): Morphological theory. An introduction to word structure in generative grammar. Oxford: Blackwell.

Williams, Edwin (1981): "On the Notions 'Lexically Related' and 'Head of a Word"'. Linguistic Inquiry 12/2: 245-274.

Williamson, Kay/Blench, Roger (2000): “The Niger-Congo languages: A classification and description of Africa's largest language". In: Heine, Bernd/Nurse, Derek (eds.): African Languages - An Introduction. Cambridge, Cambridge University: 11-42.

Zwanenburg, Wiecher (1991) : « Dépendances et hiérarchie en morphologie lexicale ». L'Information Grammaticale 50 : 38-42.

\begin{tabular}{llll}
\multicolumn{2}{l}{ Abréviations } & & \\
A & Adjectif & ACC & Accompli \\
ADV & Adverbe & CON & Connecteur \\
CNJ & Conjonction & COP & Copule \\
DEF & Défini & HAB & Habituel \\
IMP & Impératif & INACC & Inaccompli \\
LOC & Locatif & MOD & Modal \\
N & Nom & n.p. & Nom propre \\
OBJ & Objet & POSS & Possessif \\
POST & Postposition & PL & Pluriel \\
SG & Singulier & V & Verbe
\end{tabular}

\footnotetext{
18 Nos abréviations s'inspirent du modèle proposé par Chrisitna Lehmann (1980): Institut für Sprachwischenchaft Universität Köln, Arbeitspapier 37.
} 